\title{
Using a head-mounted camera to infer attention direction
}

\author{
Clara Schmitow,' Gunilla Stenberg,' \\ Aude Billard, ${ }^{2}$ and Claes von Hofsten'
}

(S)AGE

\begin{abstract}
A head-mounted camera was used to measure head direction. The camera was mounted to the forehead of 20 6- and 20 I2-month-old infants while they watched an object held at II horizontal $\left(-80^{\circ}\right.$ to $\left.+80^{\circ}\right)$ and 9 vertical $\left(-48^{\circ}\right.$ to $\left.+50^{\circ}\right)$ positions. The results showed that the head always moved less than required to be on target. Below $30^{\circ}$ in the horizontal dimension, the head undershoot of object direction was less than $5^{\circ}$. At $80^{\circ}$, however, the undershoot was substantial or between $10^{\circ}$ and $15^{\circ}$. In the vertical dimension, the undershoot was larger than in the horizontal dimension. At $30^{\circ}$, the undershoot was around $25 \%$ in the downward direction and around $40 \%$ in the upward direction. The size of the undershoot was quite consistent between conditions. It was concluded that the head-mounted camera is a useful indicator of horizontal looking direction in a free looking situation where the head is only turned moderately from a straight ahead position.
\end{abstract}

\section{Keywords}

attention, head direction, head-mounted camera, infants

Attention direction reflects a number of important psychological functions including perception, cognition and emotion. Thus, knowledge of attention direction opens up a window to a person's mind. It is especially important for learning about preverbal children who cannot yet report on their experiences. An important question in developmental psychology today is which methods can help researchers to study direction of attention in everyday situations.

Information of attention direction is revealed through a person's looking behaviour. The techniques for measuring attention direction through looking have recently been greatly improved and looking direction can now be determined within a fraction of a degree of visual angle at high frequency. The use of corneal reflection eye tracking to investigate perceptual, attentional or cognitive development in infancy is becoming increasingly more common (Gredebäck, Johnson, \& von Hofsten, 2010). In the most widely-used form of this eye tracking method, the infant sits in front of a screen where video clips or a set of still pictures are presented while the infant's direction of looking is recorded (Aslin \& McMurray, 2004; Gredebäck et al., 2010; Wu \& Kirkham, 2010). One advantage of such a system is that the infant's head does not need to be in a predetermined position, but can move rather freely within measurement space. However, a shortcoming of such devices is that the infant cannot move around but has to stay in front of a screen on which looking-direction is recorded, with a rather restrictive field of view. In real life, the child gains knowledge by looking around at the surroundings (e.g., Thelen \& Smith, 1994). Thus, methods are needed for the study of how infants direct their attention when they are active, and exploring the physical and social world.

\section{Head-mounted gaze-trackers}

There are several such methods around. One such method measures gaze direction with corneal reflection and relates it to the field of view of a scene camera (Corbetta, Guan, \& Williams, 2012; Franchak \& Adolph, 2010; Franchak, Kretch, Soska, \& Adolph, 2011). These devices allow the child to move around while their gaze is being recorded, which makes it possible to track where the child is looking while the child is interacting with his or her environment. However, a disadvantage of these methods is that they require gaze direction to be recorded in the context of a scene camera. Thus, the infant can see the eye tracking device and try to remove it (see Corbetta et al., 2012, for a similar discussion; also Aslin, 2012). Furthermore, head-mounted eye-trackers that are connected to a computer with a wire may restrict the infants' opportunities to move around (see Franchak et al., 2011 for a similar discussion). This drawback is reduced if the device is equipped with a battery that is embedded in a backpack or vest worn by the child (Franchak et al., 2011). However, the whole set then becomes heavier to carry. Thus, for younger infants, this method may not be optimal.

\section{Head-mounted cameras without eye-trackers}

Another solution is to use a head-mounted camera without an eyetracker. A head-mounted camera provides videos from the infant's field of view and where in that field the head is pointing, but information about the infant's eye-movements is not provided. However, such a device may be a good solution in studies when

\footnotetext{
1 Uppsala University, Uppsala, Sweden

${ }^{2}$ Swiss Federal Institute of Technology - EPFL, Lausanne, Switzerland

Corresponding author:

Gunilla Stenberg, Uppsala University - Department of Psychology, Box 1225, SE-75I 42 Uppsala, Sweden.

Email: Gunilla.Stenberg@psyk.uu.se
} 
detailed information regarding eye-movements is not required. The question then is: How is head direction related to looking direction?

In the present study, we investigated the possibility of using a small, light-weight video camera attached to the forehead of the infant for measuring head direction. A similar method has previously been used by Noris, Keller, and Billard (2011), Pereira, James, Jones, and Smith (2010), Smith, Yu, and Pereira (2011) and Yoshida and Smith (2008). The participating children in these studies were between 12 and 36 months of age. Videos from such a head-mounted video camera were collected from a 15 -week-old infant during activities such as sitting in a stroller or in a car-seat (see Aslin, 2009). In addition to measuring head direction, the authors pointed out the importance of "the first person view" of the surrounding that a head-mounted camera supplies; a view from the perspective body scale of the child (e.g., Yoshida \& Smith, 2008). The "first person view" has also been called "active vision" or "animated vision" in computer vision literature (e.g., Ballard, 1991). Studies in this area highlight advantages of active vision in comparison to passive vision in how intelligent systems learn about physical properties of objects (Aloimonos, Weiss, \& Bandyopadhyay, 1988; Ballard, 1991; Yu, Smith, Shen, Pereira, \& Smith, 2009). The infant's visual system is not independent from his/her motor and cognitive systems. Thus, the infant discovers new properties of objects by moving, changing perspective, and touching them. Therefore, research tools that are able to follow the infant's movements by capturing the infant's perspective are likely to lead to new insights on how infants learn about the world.

\section{The need of a calibration}

In their study, Yoshida and Smith (2008) found a head-mounted camera was useful in situations where the infant acts on objects. The authors examined if the camera could follow the direction of attention when the infant was presented with three stickers located at the right, the left and the midpoint of a table located in front of the infant (corresponding to a "table-top toy play"). The results indicated that the view from the head-mounted camera was closely related to attention direction, but it lagged systematically the dynamic properties of the gaze shifts. Yoshida and Smith (2008) also found that the head-mounted camera was useful in a more naturalistic context of toy play when also the infant's mother participated. Pereira and co-workers (2010) examined infant head movements while the infants examined different toys. When the infant's interest in a particular toy decreased, the toy was removed and the infant was given a new toy. Because the toys were given one at a time, the infant just had to focus on that particular toy, in a constrained visual field. Thus, neither of these studies provides information about how the head-mounted camera view corresponds to the eye gaze direction other than in a narrow field of view in front of the infant.

In order to establish the usefulness of the head mounted camera for making conclusions about where the infant is directing his/her attention, it is important to estimate the correspondence between the view captured by the head-mounted camera and where the infant is looking.

\section{Eye-head coordination}

To assess the usefulness of a head-mounted camera for estimating attention direction, it is necessary to determine how tightly head movements and looking are coupled in everyday looking tasks. Except for small fixation-shifts, all changes in attention direction involve both the eyes and the head (Bizzi, 1981; von Hofsten, Vishton, Spelke, Feng, \& Rosander, 1998). Therefore, measurements of head movements could, in principle, be used to infer attention direction. The obvious weakness with using head direction to infer attention direction is the inertia of the head, which lags the eyes when shifting attention. Thus, in shifting gaze, the eyes move more rapidly than the head. However, the head catches up and in the end the two body parts become aligned once again (Bizzi, 1981).

The development of eye-head coordination has an early onset. From around 4 months of age, infants use extensive head movements when tracking objects moving horizontally in front of them. The head movements lag the object by about $0.3-0.4 \mathrm{~s}$, but in all other respects, they are well coordinated with the eye movements and the gaze attaining almost unity gain (von Hofsten \& Rosander, 1997). Von Hofsten et al. (1998) used head movements to investigate 6-month-old infants' visual tracking of an object moving on a large vertical surface. They found that head and eyes moved together in tracking the object and that the head movements were well geared to the amplitude of the object motion. Von Hofsten, Feng, and Spelke (2000) and Jonsson and von Hofsten (2003) found that head movements could also be used to estimate predictive gaze shifts to the reappearance of a temporarily occluded moving object. Both these studies found that the head started to move toward the reappearance position before the object arrived there.

Although head movements reflect looking-behaviour very well in these situations, an eye-tracking study by Grönqvist, Gredebäck, and von Hofsten (2006), using corneal reflection, found that horizontal tracking was superior to vertical tracking in aspects such as timing and gain in smooth pursuit. Further, Jonsson and von Hofsten (2003) found that although head movements were nicely geared to object motion at the central part of the visual field, it systematically underestimated looking direction at extreme peripheral object directions. In light of these findings, the usability of head movements as a measure of looking needs to be further examined.

The present study was designed to determine the coupling between head- and attention-direction when infants look at an interesting object presented at different vertical and horizontal positions. By also including infants younger than 12 months, i.e., 6-month-old infants, we wanted to examine the range of ages at which a headmounted camera can be used. Using this method with younger infants may enable the study of early indicators of social development such as following pointing or gaze directions of others (D'Entremont, Hains, \& Muir, 1997; von Hofsten, Dahlström, \& Fredriksson, 2005). If a head-mounted camera proves to be a reliable tool to study infants' attention direction, this will open new possibilities to the study of infant social as well as cognitive development in naturalistic situations.

\section{Method}

\section{Subjects}

Forty full-term infants participated in the study; 20 6-month-old infants ( $M=26$ weeks, $S D=1.5$ weeks; 10 girls and 10 boys) and 20 12-month-old infants ( $M=51.5$ weeks, $S D=1.7$ weeks; 10 girls and 10 boys). Ten additional infants were excluded because of infant fussiness or technical problems. All infants were recruited from birth records in a greater metropolitan area in Sweden. The participants received a gift certificate with a value of approximately 
$€ 10$. The study was approved by the Regional Ethical Review Board and accorded with the ethical standards specified in the 1964 Declaration of Helsinki.

\section{Apparatus}

A small wireless camera (WearCam; $27 \times 27 \times 38 \mathrm{~mm}$ ), developed by Piccardi and co-workers (2007), was attached to the forehead of the infants, with two headbands keeping it in place. The camera provides a diagonal field of view of 92 degrees $\left(56^{\circ}\right.$ vertical and $74^{\circ}$ horizontal) and records images of $640 \times 480$ pixels at 30 frames per second, which is essential to capture the child's head movements. An on-board rechargeable battery (45 mins' duration) was placed on the back of the head to balance the weight of the apparatus at the forehead. The weight of the whole camera, battery included, was $60 \mathrm{~g}$. The camera was designed to be worn by children between 6 months and 2 years of age (head perimeter $35-48 \mathrm{~cm}$ ). The camera sent data wireless to a computer at $2.4 \mathrm{GHz}$, where the recordings from the experiment were stored for later analyses. A blinking buzzing object was used to attract the infants' attention at predetermined vertical and horizontal positions. It consisted of a battery-powered transparent ball with a diameter of $7 \mathrm{~cm}$, and with a visual angle of about $5^{\circ}$ viewed at a distance of 85 $90 \mathrm{~cm}$. When it was activated, two sets of moving blinking lights moved rapidly inside the ball and a soft buzzing sound was heard. The toy was selected from a number of different toys tested on 6and 12-month-old infants. It attracted the looking of the infants efficiently. The whole session was also videotaped by a stationary video camera.

\section{Experimental setup}

The experiment was performed in a room equipped with a table $(60 \mathrm{~cm} \times 120 \mathrm{~cm})$, a stationary video camera, and a portable computer connected to the head-mounted camera. The computer was placed on a chair in one corner of the room to prevent the infant from seeing the computer screen. The infant was sitting in a high-chair at the table during the experiment with the eyes $90 \mathrm{~cm}$ from the floor and $80 \mathrm{~cm}$ from the opposite side of the table. The high-chair was placed at the left end of the table when measuring the head angles to the horizontal positions to the right, and at the right end of the table when measuring the head angles for the horizontal positions to the left. The location of the stationary camera was changed between these two series of horizontal positions in order to get an optimal picture of the infant. The experimenter was seated on a movable chair at the opposite side of the table. The object positions were indicated at the side of the table, close to the experimenter, and thus invisible to the infant. The stimulus object was presented at 11 horizontal positions. The horizontal positions were divided into two sets of presentations, one for straight ahead and positions to the right (the positive angles), and one for straight ahead and positions to the left (the negative angles). The horizontal positions were situated at $-80^{\circ},-50^{\circ},-40^{\circ},-30^{\circ},-20^{\circ}, 0^{\circ}, 20^{\circ}$, $30^{\circ}, 40^{\circ}, 50^{\circ}$ and $80^{\circ}$ from a straight-ahead position. At each position the blinking, buzzing object was shown for $2-3 \mathrm{~s}$, during which time the experimenter made sure that the infant was looking at the object. In the horizontal condition, the experimenter's face was situated behind the object, and from there she could easily judge whether the subject was fixating on the target. Between presentations, the experimenter moved along the opposite side of the table

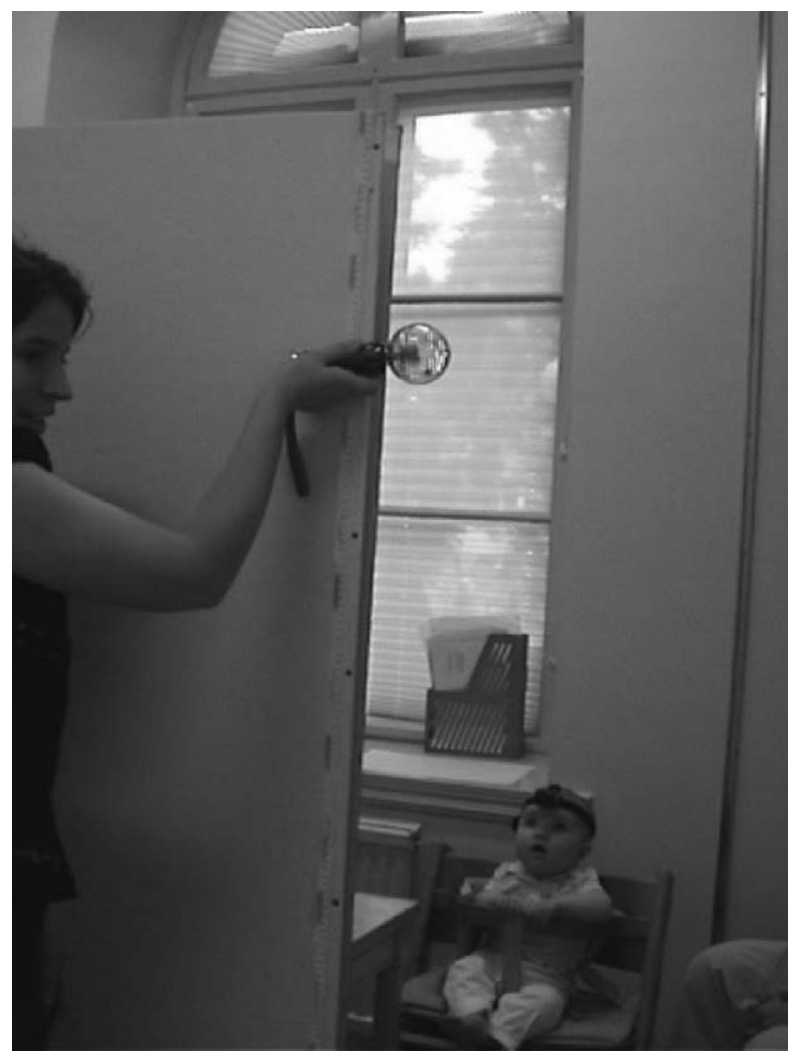

Figure I. Picture of the setting in the vertical plane.

on the movable chair until reaching the next object position where the attractive toy was shown. When the most peripheral position had been tested, the series of test positions was run in reverse order.

The infant was sitting in the high-chair at the table also in the vertical condition. A paper screen was placed at the opposite side of the table, between the experimenter and the infant. The edge of this screen was located straight in front of the centre of the high-chair, $80 \mathrm{~cm}$ from the infant's eyes. The stimulus object was presented at 9 vertical positions. The object positions were marked on the backside of the screen, invisible to the infant. These positions were also divided into two sets of presentations, one for straight ahead and positions up (the positive angles), and one for straight ahead and positions down (the negative angles). The vertical positions were situated at $-48^{\circ}$ (close to the floor), $-40^{\circ},-30^{\circ},-20^{\circ}$, $0^{\circ}, 20^{\circ}, 30^{\circ}, 40^{\circ}$ and $50^{\circ}$ from straight ahead. When the most peripheral position had been tested, the series of test positions was run in reverse order. In the vertical condition, it was not possible to position the experimenter straight behind the target, and therefore she showed the attractive target without disclosing herself (see Figure 1).

The infants were either presented with the horizontal or the vertical positions first. In each condition, half of the subjects were presented with the positive angles first, and half with the negative angles first. The order of the blocks was randomly determined. Within each block, a ladder procedure was adopted. That is, $0^{\circ}$ was always presented first and thereafter the other angles were presented in an incremental order until the most peripheral position (the largest angle) had been tested. Thereafter, the positions were shown in reverse order with $0^{\circ}$ being the last presentation. The presentations at $0^{\circ}$ were used as calibrations for the sequence of trials, 
and the fact that $0^{\circ}$ was presented both at the beginning and the end of the series, made it possible to determine whether the position of the head relative to the stimulus object had changed.

\section{Experimental procedure}

To begin with, the accompanying parent was informed about the procedure and was told that she/he could interrupt the experiment at any time. The experimenter explained how to attach the small camera to the infant's forehead. Thereafter, the parent was asked to sign a consent form. When the infant was sitting comfortably in the high-chair, the parent was asked to put on the camera. Meanwhile, the experimenter was holding and playing with an attractive toy (distractor) in front of the infant. When the camera was attached, the experimenter adjusted the camera to make sure that it was pointing straight ahead and that the target toy was seen in the middle of the screen when the infant looked at it. The whole experiment, including the mounting of the camera, was completed in approximately 10 mins. During the experiment, the parent was sitting in a chair behind the infant's high-chair.

\section{Measurement of head direction as an indicator of attention direction}

For each object position, the view of the head-mounted camera showed where the head was directed relative to the attractive fixation-object. First, a mark was made on the video screen when the head was directed at $0^{\circ}$. At this point, it was assumed that attention was directed at the attracted object. The $0^{\circ}$ fixation was checked at several times during the experiment. The deviation of the head direction from this position on the video screen was then measured for each object position. This deviation reflects the difference between head direction and object location. The video data were analyzed manually second by second in a video program (Vegas 7.0). In this program, the coder watched the object on the screen and marked its position with a cross. When the object was straight in front of the infant at $0^{\circ}$ (the calibration point), a red cross was created over the object on the video screen. For the other angles (different than $0^{\circ}$ ), black crosses were created over the object to signal the deviation of the head-direction from object location at these points. This analysis is based on the assumption that the infant looks at the toy that is blinking. At the horizontal positions, the experimenter's face was hidden behind the toy; in the vertical positions this was not possible and the experimenter was not visible at all. It should be pointed out that no other possible distractions were present in the visual field. The external camera-assessments and the low variability in the data indicated that the infants looked at the attractive toy. All data were thus related to the calibration-position of the object. The positional differences between the calibration point and the object positions were measured in $\mathrm{mm}$ on the video screen and then converted into degrees. This study was designed to measure head direction, and therefore, the data did not provide information about the time course of errors between head direction and attention-direction. A second coder scored $20 \%$ of the data (differences in $\mathrm{mm}$ between calibration point and object positions) using the same video program (Vegas 7.0). Correlations between the primary and the secondary coder ranged from .78 to $.98(M=.86)$ for horizontal positions to the right and from .93 to $.99(M=.95)$ for horizontal positions to the left. In the vertical positions, the correlations ranged from .79 to $.97(M=.89)$ for vertical positions up, and from .75 to $.95(M=.88)$ for vertical positions down. All correlations were significant (with $p$ values ranging from .000 to .032). Children's heads are not as stable as adults', thus there was some variability between measurements. The videos from the stationary camera were coded by a third coder to determine whether the infants were looking at the blinking object at each horizontal and vertical position. Cline (1967) found, for instance, that the standard deviation of adults' judgements of gaze direction was $2^{\circ}$ to $3.5^{\circ}$. This was enough for our purpose, as the object was about 5 degrees of diameter. A fourth coder independently coded $25 \%$ of these videos with $100 \%$ agreement.

\section{Results}

\section{Comparing head and object direction}

Pearson correlations were performed to test the coupling between head direction and the direction to an attractive object in the visual field. Furthermore, the coupling between object eccentricity and head direction was analyzed. There was a high correspondence between head direction and direction to the object in the horizontal plane (see Figure 2). For the 6-month-old infants, the Pearson product-moment correlations were .94 for the positions to the right and .97 for the positions to the left, and for the 12-month-old infants, they were .96 for the positions to the right and .95 for the positions to the left. The discrepancy between head direction in the horizontal dimension and object direction is shown in Table 1. The correspondence was more modest in the vertical plane (see Figure 3). For the 6-month-old infants, the correlation was .82 for the upward positions and .91 for the downward positions. The discrepancy between head direction in the vertical dimension and object direction is shown in Table 2.

For the 12-month-old infants, the correlations were .84 for the upward positions and .86 for the downward positions. The confidence intervals of the correlation coefficients for the horizontal positions did not overlap with the confidence intervals of the vertical positions; this indicates that the performance for the horizontal positions were significantly different from the vertical ones. Linear equations corresponding to each of the correlations are shown in Table 3. It shows that the deviation from perfect correspondence between head and object directions is proportional to the deviation from straight ahead, that is, the deviation from perfect correspondence at $40^{\circ}$ to the side is twice as large as the deviation at $20^{\circ}$. At the extreme positions, the deviations are larger than proportional which is an indication that at those positions, the head does not make a complete turn but instead the eyes turns to the side to fixate the object. This is in accordance with the results of Jonsson and von Hofsten (2003). The deviations from a perfect head and eye correspondence were calculated by subtracting object direction from head direction. To study possible effects of age, four ANOVAs were performed with the deviations in the horizontal plane for the right and left, and in the vertical plane, up and down. Different ANOVAs were performed for each direction as the experiment was divided in four different, randomized sections. The mixed design ANOVA with age as between-subjects variable showed no effect of age, $F(1,38)=1.38, p=.25$ (right), $F(1,37)<1.0$ (left), $F(1,37)<1.0$ (up), $F(1,35)=2.80, p=.10$ (down). Thus, the 6- and 12-month-old infants performed similarly.

\section{Discussion}

The objective of the current study was to determine the coupling between head and attention direction, using a head-mounted 


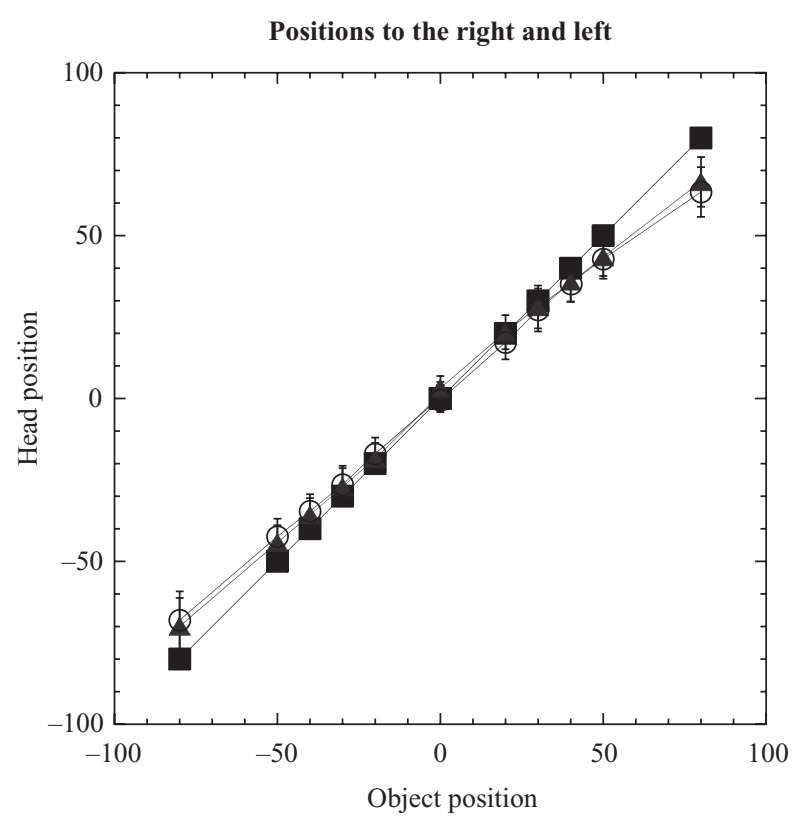

Figure 2. The average position of the head (measured by the head-camera in degrees) for the 6-and 12-month-old infants when they fixate on the object in the horizontal positions. Squares denote object position. Triangles show head position for the 6-month-old infants and circles show head position for the 12-month-old infants. Error bars show the confidence intervals.

Table I. Mean head direction and standard error relative to straight ahead for the horizontal positions (right/left).

\begin{tabular}{lcccccc}
\hline & \multicolumn{7}{c}{ Difference in the angle relative to straight ahead } \\
\cline { 2 - 8 } & 0 & 20 & 30 & 40 & 50 & 80 \\
\hline Rightward angles & & & & & \\
6 months: & 3.23 & 0.39 & -1.84 & -4.57 & -6.47 & -13.48 \\
Mean (SE) & $(0.92)$ & $(1.29)$ & $(1.62)$ & $(1.41)$ & $(1.47)$ & $(1.89)$ \\
I2 months: & -.47 & -2.76 & -2.82 & -4.47 & -7.22 & -16.61 \\
Mean (SE) & $(.92)$ & $(1.288)$ & $(1.620)$ & $(1.408)$ & $(1.467)$ & $(1.891)$ \\
Leftward angles & & & & & & \\
6 months: & 1.24 & 1.53 & 2.92 & 4.33 & 5.73 & 10.07 \\
Mean (SE) & $(.95)$ & $(1.18)$ & $(1.40)$ & $(1.26)$ & $(1.32)$ & $(2.14)$ \\
I2 months: & .343 & 3.08 & 4.27 & 6.03 & 7.82 & 12.39 \\
Mean (SE) & $(.98)$ & $(1.21)$ & $(I .44)$ & $(1.29)$ & $(1.35)$ & $(2.20)$ \\
\hline
\end{tabular}

camera, when 6- and 12-month-old infants were looking at an attractive object presented at different horizontal and vertical positions in the visual field. Head and eyes are tightly coupled in everyday looking, and by measuring head movements it is therefore possible to get an indication of where the child is looking (see Jonsson \& von Hofsten, 2003). The results show that egocentric head direction is systematically related to object direction. The object that the infant was looking at was visible in the head-mounted camera view at all locations. However, the results indicated that the head did not turn as much as required to be directed at the target. This was valid for both ages. In the horizontal plane, head direction accounted for $88 \%$ of object direction, and in the vertical plane, head direction accounted for about two-thirds of object direction.

Although the correlations between object direction and head direction were quite high irrespective of age (around .95 for the

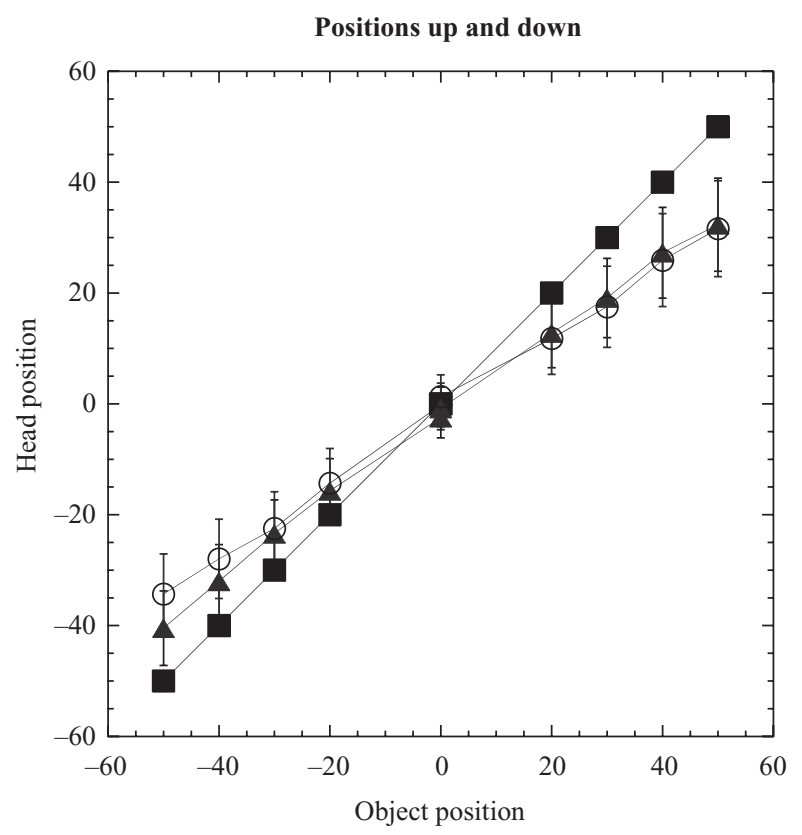

Figure 3. The average location of the head compared to the position of the object for the 6- (triangles) and 12-month-old infants (circles) in the vertical conditions. Squares denote object position. Error bars show the confidence intervals.

Table 2. Mean head direction and standard error relative to straight ahead for the vertical positions (up/down).

\begin{tabular}{lccccc}
\hline & \multicolumn{5}{c}{ Difference in the angle relative to straight ahead } \\
\cline { 2 - 6 } & 0 & 20 & 30 & 40 & 50 \\
\hline Upward angles & & & & & \\
6 months: & -.82 & -7.17 & -10.87 & -12.72 & -17.67 \\
Mean (SE) & $(.95)$ & $(1.56)$ & $(1.76)$ & $(2.02)$ & $(2.08)$ \\
I2 months: & 1.07 & -7.81 & -12.46 & -14.07 & -18.46 \\
Mean (SE) & $(.97)$ & $(1.60)$ & $(1.81)$ & $(2.07)$ & $(2.13)$ \\
Downward angles & & & & & \\
6 months: & -2.47 & 4.35 & 6.56 & 8 & 9.55 \\
Mean (SE) & $(.90)$ & $(1.42)$ & $(1.50)$ & $(1.63)$ & $(1.66)$ \\
I2 months: & -1.563 & 6.43 & 7.99 & 12.54 & 15.85 \\
Mean (SE) & $(.97)$ & $(1.55)$ & $(1.63)$ & $(1.77)$ & $(1.80)$ \\
\hline
\end{tabular}

horizontal positions), and the relation was approximately linear as shown in Table 3, it can be noted from Table 3 that the linear equations relating head and object direction are more similar between the two ages than between the vertical and horizontal planes. The linear trends reflect that the eyes turned proportionally more than the head. At the larger angles, the undershoot of the head was, however, more than proportional to the direction of the object.

The validity of the measures presented here is based in the assumption that the infants were in fact looking at the object. Different factors indicated that this was the case. First, the experimenter did not move to the next location if the infant did not look at the toy (and it is easy for the experimenter to discern whether the infant is looking at the toy or somewhere else). It is of course possible that the infant fixated on parts of the experimenter's face other than the middle where the attractive object was placed, but as mentioned 
Table 3. The linear equations corresponding to the correlations between head position and object position in the horizontal and vertical planes for the 6- and the 12 month-old infants.

\begin{tabular}{llll}
\hline R Squares & & \multicolumn{1}{c}{6 months } & 12 months \\
\hline Horizontal plane & Right & $y=4.05+0.786 x$ & $y=1.63+0.799 x$ \\
& Left & $y=0.014+0.883 x$ & $y=-0.081+0.853 x$ \\
Vertical plane & Up & $y=-0.72+0.674 x$ & $y=0.437+0.615 x$ \\
& Down & $y=-1.49+0.761 x$ & $y=-1.841+0.655 x$ \\
\hline
\end{tabular}

above, the judgment where another person is looking is very good (Cline, 1967; Gibson \& Pick, 1963). The low variability of the data also strengthens this assumption. In addition, the differences found here cannot be explained by the head-mounted camera sliding on the head, as pilot studies showed that the camera did not slip when it was used.

The blinking, buzzing object was shown at predetermined reference positions. The deviation of the head from those positions was measured. In a situation where a head-mounted camera is used to get an estimate of where the child is looking, however, there are no reference directions from which the head deviation can be calculated. The camera only provides information of the discrepancy between head direction and object location. This is shown in Tables 1 and 2. In a natural situation where a subject looks around freely, the head rarely points far to the side. If something happens in the periphery, the eyes might initially turn more extensively, but this is usually followed by a turning of the head and the body in the direction of the event. Furthermore, infants are known to follow object trajectories to a greater extent with their heads than adults do (von Hofsten et al., 2000). If the head deviates $30^{\circ}$ or less from straight ahead in the horizontal plane, the obtained angular differences between head and object directions were close to $3^{\circ}$ for the 6-month-old infants and slightly over $4^{\circ}$ for the 12-month-old infants. In the vertical plane, however, the discrepancy between head direction and attention direction is larger and the measurement of head direction is therefore less informative about where the infant is actually looking. The random errors were small, but the systematic errors were large, which indicates that the eye movements constituted a larger proportion of the gaze shifts in this condition.

To sum up, by examining the coupling between head direction and attention direction in the horizontal and vertical planes, we have provided information concerning the applicability of a headmounted camera to measure the young child's focus of attention. Our results show that looking can be inferred from measurements of head direction, especially when an object is presented in the horizontal plane. Our results are in line with those reported by Jonsson and von Hofsten (2003), showing that the correspondence between head and gaze direction was higher for positions within the central visual field of the infant than for the most extreme positions in the horizontal and vertical planes. Thus, while the camera was found to be a good proxy of the infant's attention at small horizontal angles, researchers should be aware of the deviations associated with the more extreme horizontal and vertical positions.

The results show that the coupling between head direction and attention direction is similar at the two ages. Thus, despite some limitations, we found the head-mounted camera to be a useful method to capture 6- and 12-month-old infants' visual experiences in the present context. Pereira and co-authors (2010) have reported that 12-month-old infants tolerate a head-mounted camera. In this study, we have expanded the age range at which a head-mounted camera can be used. We found the device to be tolerated also by infants as young as 6 months. A crucial aspect of the present method is placing the camera on the infant's head. In the current study, the parents were asked to attach the camera to the infant's forehead while the experimenter distracted the infant with a toy. We found this procedure to be very effective in getting the infants to accept the camera. After the camera had been attached, most infants soon forgot about wearing the device and paid no further attention to it.

In contrast to head-mounted cameras used in other studies (e.g., Yoshida \& Smith, 2008), the camera we used was wireless. Although the infant may turn his or her head without any major restrictions while wearing a camera with a wire, such a camera might be best suited in situations when the child is sitting (which young children and specially infants do a lot during face-to-face interaction with a parent and during solitary play). However, a wireless camera allows for the child to move around freely. Accordingly, without a wire, a head-mounted camera allows for studies in far more domains; the room will no longer set bounds to the object of study. A next step could be to test the head-mounted camera in situations where the infant is crawling or walking.

One important difference between using a head-mounted camera and a traditional stationary camera to record where the child is directing her/his attention is that a head-mounted camera gives a first-person view of the situation; the view from the child's perspective. When the view captured by a stationary camera is examined, this examination is based on the outside observer's subjective interpretation of what the infant sees. Apart from the fixated object, other objects in the visual periphery are shown that might attract looking in the moments to come. With just stationary cameras it is harder to interpret the visually-inspired actions of the child. As mentioned above, the visual system works together with the motor and cognitive systems; some examples of this is how infants learn about size or shape of objects by manipulating and looking at them (Yu et al., 2009) and how infants look at objects just before they perform an action on them (Rosander \& von Hofsten, 2011). Thus, a tool that captures the infant's view of objects and people in real situations can give us more information about how the young child sees, manipulates and learns about the world.

\section{Conclusions}

A head-mounted camera is undoubtedly a cruder method for estimating attention direction than conventional and mobile eye trackers. Compared to head-mounted eye-trackers, however, the simplicity of the method is very attractive. It is a good and very low-cost solution for estimating looking in naturalistic situations. It also provides a "first person" view of the surroundings. Researchers interested in using head-mounted cameras should, however, take into considerations that when the head is turned in a large eccentric direction, the head direction greatly underestimates looking direction. This problem could be avoided if a scene camera is used to determine whether the child is turning the head very much and exclude those passages from the data. In a naturalistic situation, the head is rarely turned more than $30^{\circ}$ in the horizontal direction without turning the body, and then a head-mounted camera can be used with confidence. Random error is not a great problem as can be seen from the small variance in the data. Experiments using this method should also be aware of the larger 
systematic errors in the vertical dimension. Furthermore, as has been found in an earlier study (Yoshida \& Smith, 2008), a headmounted camera is not suitable for measuring the timing of looking-responses. The next step will be to test the camera in everyday situations in which the infant interacts with other people. This will broaden the scope of situations in which this method may be used. Future studies should also test the camera on adults.

\section{Acknowledgments}

We would like to thank all parents and infants for their participation in this research.

\section{Funding}

This project was funded by the EU Commissions Framework programmes NEST (project 15636).

\section{References}

Aloimonos, J., Weiss, I., \& Bandyopadhyay, A. (1988). Active vision. International Journal of Computer Vision, 1(4), 333-356.

Aslin, R. N. (2009). How infants view natural scenes gathered from a head-mounted camera. Optometry and Vision Science, 86(6), $561-565$.

Aslin, R. N. (2012). Infant eyes: A window on cognitive development. Infancy, 17(1), 126-140.

Aslin, R. N., \& McMurray, B. (2004). Automated corneal-reflection eye-tracking in infancy: Methodological developments and applications to cognition. Infancy, 6(2), 155-163.

Ballard, D. H. (1991). Animated vision. Artificial Intelligence, 48, 57-86.

Bizzi, E. (1981). Eye-head coordination. In V. B. Brooks (Ed.), Handbook of Physiology, 3 (pp. 1321-1336). Bethesda, MD: American Physiological Society.

Cline, M. G. (1967). The perception of where a person is looking. American Journal of Psychology, 80, 41-50.

Corbetta, D., Guan, Y., \& Williams, J. L. (2012). Infant eye-tracking in the context of goal-directed actions. Infancy, 17(1), 102-125.

D'Entremont, B., Hains, S. M. J., \& Muir, D. W. (1997). A demonstration of gaze following in 3- to 6-month-olds. Infant Behavior and Development, 20(4), 569-572.

Franchak, J. M., \& Adolph, K. E. (2010). Visually guided navigation: Head-mounted eye-tracking of natural locomotion in children and adults. Vision Research, 50(24), 2766-2774.

Franchak, J. M., Kretch, K. S., Soska, K. C., \& Adolph, K. E. (2011). Head-mounted eye tracking: A new method to describe infant looking. Child Development, 82(6), 1738-1750.

Gibson, J. J., \& Pick, A. D. (1963). Perception of another person's looking behavior. American Journal of Psychology, 76(3), 318-394.
Gredebäck, G., Johnson, S., \& von Hofsten, C. (2010). Eye tracking in infancy research. Developmental Neuropsychology, 35(1), 1-19.

Grönqvist, H., Gredebäck, G., \& von Hofsten, C. (2006). Developmental asymmetries between horizontal and vertical tracking. Vision Research, 46(11), 1754-1761.

Jonsson, B., \& von Hofsten, C. (2003). Infants' ability to track and reach for temporarily occluded objects. Developmental Science, 6(1), 86-99.

Noris, B., Keller, J.-B., \& Billard, A. (2011). A wearable gaze tracking system for children in unconstrained environments. Computer Vision and Image Understanding, 115(4), 476-486.

Pereira, A. F., James, K. H., Jones, S. S., \& Smith, L. B. (2010). Early biases and developmental changes in self-generated object views. Journal of Vision, 10(11):22, 1-13.

Piccardi, L., Noris, B., Barbey, O., Billard, A., Schiavone, G., Keller, F., \& von Hofsten, C. (2007, August). WearCam: A head mounted wireless camera for monitoring gaze attention and for the diagnosis of developmental disorders in young children. In Proceedings of the 16th IEEE International Symposium on Robot \& Human Interactive Communication.

Rosander, K., \& von Hofsten, C. (2011). Predictive gaze shifts elicited during observed and performed actions in 10-month-old infants and adults. Neuropsychologia, 49(10), 2911-2917.

Smith, L. B., Yu, C., \& Pereira, A. F. (2011). Not your mother's view: The dynamics of toddler visual experience. Developmental Science, 14(1), 9-17.

Thelen, E., \& Smith, L. B. (1994). A dynamic systems approach to the development of cognition and action. Cambridge, MA: MIT Press.

von Hofsten, C., Dahlström, E., \& Fredriksson, Y. (2005). 12-month-old infants' perception of attention direction in static video images. Infancy, 8(3), 217-231.

von Hofsten, C., Feng, Q., \& Spelke, E. S. (2000). Object representation and predictive action in infancy. Developmental Science, 3(2), 193-205.

von Hofsten, C., \& Rosander, K. (1997). Development of smooth pursuit tracking in young infants. Vision Research, 37(13), 1799-1810. von Hofsten, C., Vishton, P., Spelke, E. S., Feng, Q., \& Rosander, K. (1998). Predictive action in infancy: Tracking and reaching for moving objects. Cognition, 67(3), 255-285.

Wu, R., \& Kirkham, N. Z. (2010). No two cues are alike: Depth of learning during infancy is dependent on what orients attention. Journal of Experimental Child Psychology, 107(2), 118-136.

Yoshida, H., \& Smith, L. B. (2008). What's in view for toddlers? Using a head camera to study visual experience. Infancy, 13(3), 229-248.

Yu, C., Smith, L. B., Shen, H., Pereira, A. F., \& Smith, T. G. (2009). Active Information Selection: Visual Attention Through the Hands. IEEE Transaction on Autonomous Mental Development, 1(2), 141-151. 